\title{
Field-emission characteristics of conical boron nitride nanorods
}

\author{
H Z Zhang ${ }^{1}$, Q Zhao ${ }^{2}$, J Yu ${ }^{1}$, D P Yu ${ }^{2}$ and Y Chen ${ }^{1}$ \\ ${ }^{1}$ Department of Electronic Materials Engineering, Research School of Physical Sciences and Engineering, \\ Australian National University, Canberra, ACT, 0200, Australia \\ ${ }^{2}$ State Key Laboratory for Mesoscopic Physics, Electron Microscopy Laboratory, School of Physics, Peking \\ University, Beijing 100871, People's Republic of China \\ E-mail: hongzhou.zhang@anu.edu.au
}

Received 16 October 2006, in final form 1 November 2006

Published 15 December 2006

Online at stacks.iop.org/JPhysD/40/144

\begin{abstract}
We present the electron field-emission (FE) characteristics of conical boron nitride nanorods grown on a (1 $\left.\begin{array}{lll}0 & 0\end{array}\right)$ n-type silicon substrate. The emission current can be up to $\sim 60 \mu \mathrm{A}$ at an applied voltage of $\sim 3 \mathrm{kV}$. Two distinct slopes are evident in the Fowler-Nordheim (FN) plot. The FE characteristics can be explained using a site-related tunnelling-controlled mechanism. The occurrence of two FN slopes is attributed to the switchover from tip emission to side emission, which results from the differences in interface barrier, geometry, as well as the total emission area of the two emission interfaces.
\end{abstract}

\section{Introduction}

Boron nitride (BN) has many merits as the cold electron field emitter. It has negative electron affinity, high mechanical and chemical stability, low dielectric constant and effectively doping capability $[1,2]$. The advantages of BN-based emitters have been documented [3-6]; for example, field-emission (FE) characteristics of the conventional Si emitter can be substantially improved through BN coating [3]. To achieve better FE performance, on the other hand, quasi-one-dimensional (1D) materials exemplified by the carbon nanotube [7] have been studied extensively because of their small sizes and thus large field-enhancement factors. Nanosized BN emitters have been explored recently. Experiments reveal that nanofilms of hexagonal polycrystalline $\mathrm{BN}$ exhibit a reduced potential barrier [4] and BN nanotubes show stable electron FE current [8]. It has also been proposed theoretically that the $\mathrm{BN}$ nanocone has a reduced work function [9]. Furthermore, 1D BN nanostructures are found to exhibit significant resistance to oxidation at high temperatures [10]. Therefore, the BN nanorod consisting of stacking cones might be a promising candidate for the field emitter. Recently, we have grown conical BN nanorods on a silicon substrate by using a ball-milling and annealing method [11-13]. In this paper, we report FE characteristics of the conical BN nanorods.

\section{Experimental}

The BN nanorods were grown on a (100) n-type silicon substrate (electrical resistivity $2-9 \Omega \mathrm{cm}$ ) via a ball-milling and annealing method. Boron carbide powder was first milled in nitrogen $(300 \mathrm{kPa})$ for $100 \mathrm{~h}$ at room temperature. The milled powder was then loaded into an alumina combustion boat. The silicon substrate coated with a layer of ferric nitrate was placed directly on the milled powder. They were annealed in nitrogen at $450{ }^{\circ} \mathrm{C}$ for $1 \mathrm{~h}$ and then at $1300^{\circ} \mathrm{C}$ for $8 \mathrm{~h}$. The nanorods were found on the surface of the silicon substrate. The growth technique is also detailed elsewhere [11-13]. The morphology and structure of as-grown nanorods were studied by using a Hitachi S-4500 FE scanning electron microscope (SEM) and a Philips CM300 transmission electron microscope (TEM) working at $300 \mathrm{kV}$. The composition of the nanorods was identified by using an x-ray energy dispersive spectrometer (XEDS), fitted with a super-ultra-thin window. For FE measurement, a twoparallel-plate configuration was employed in a vacuum chamber evacuated to a pressure below $\sim 5 \times 10^{-7} \mathrm{~Pa}$. The sample attached to a stainless-steel plate was biased as cathode with the other stainless-steel plate as anode. The emission current was measured using a Keithley 485 picoammeter.

\section{Results and discussion}

A low-magnification SEM image of the interface morphology of the silicon substrate shows that a large number of randomly oriented nanorods cover the silicon surface with a coverage of about 10 nanorods per $\mu \mathrm{m}^{2}$ (figure $1(a)$ ).

The area with such coverage usually extends over $10^{3}$ $\mu \mathrm{m}^{2}$. The nanorods are $\sim 10 \mu \mathrm{m}$ long. The diameters of $\sim 300$ nanorods were measured from the SEM image and a histogram 
(a)

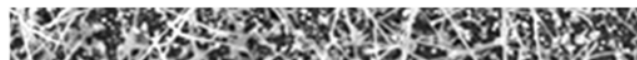

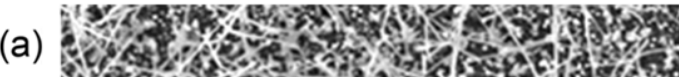
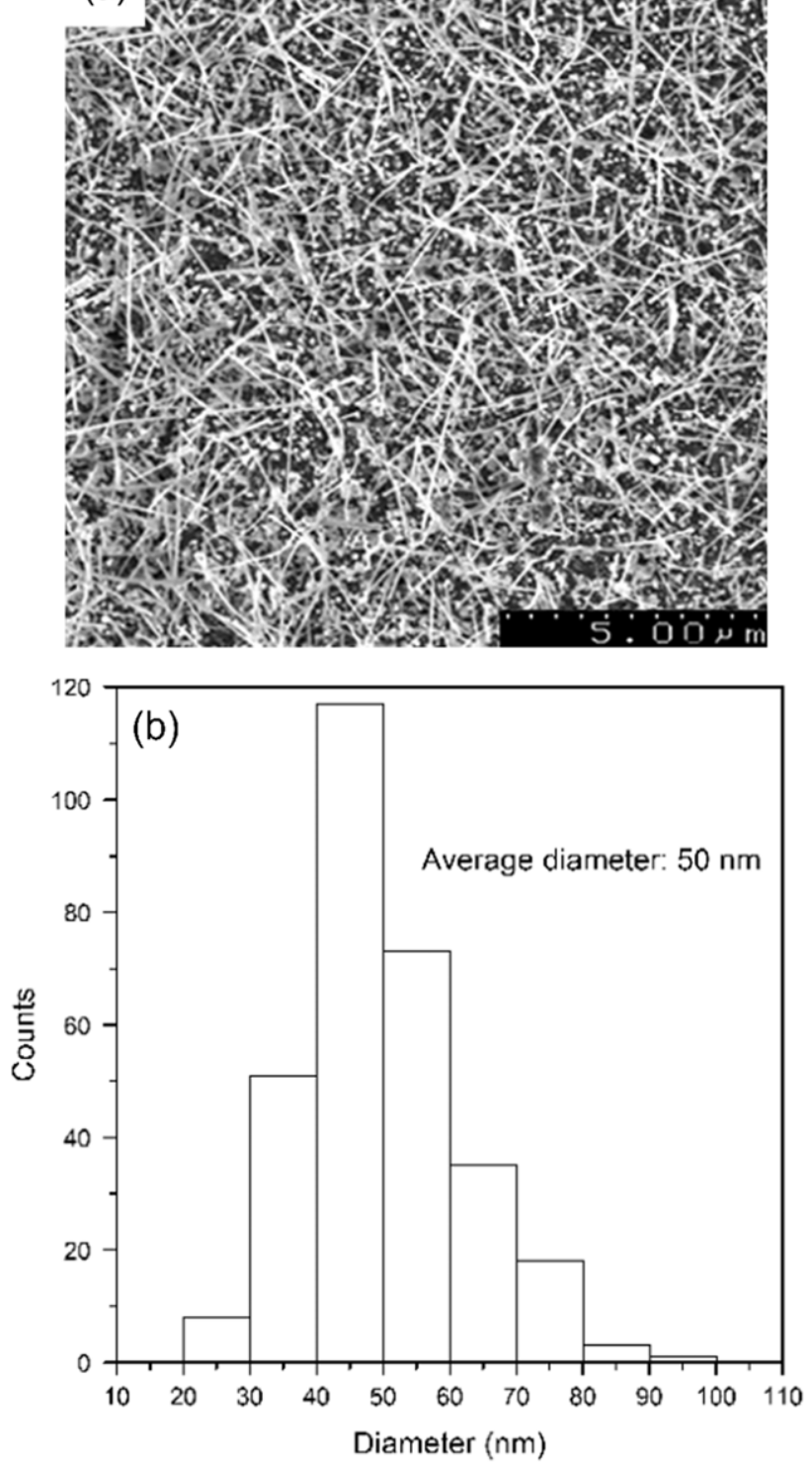

Figure 1. (a) SEM image showing a large number of nanorods grown on the Si surface. (b) Histogram of the nanorod diameters revealing an average diameter of $50 \mathrm{~nm}$.

of the diameter distribution is depicted as in figure $1(b)$. The average diameter of the nanorods is $\sim 50 \mathrm{~nm}$ with a standard deviation of $\sim \pm 30 \mathrm{~nm}$.

Figure $2(a)$ is a bright-field TEM image of typical nanorods. It can be seen that the nanorods have conical tips and bulbous attachments. X-ray energy dispersive spectra of the nanorods and the attachments (not shown here) demonstrate that they are $\mathrm{BN}$ and $\mathrm{Fe}-\mathrm{Si}$ alloy, respectively [11-13]. The microstructures of the nanorod tips and side surfaces are of special importance to their FE properties. Figure 2(b) shows the lattice image of a nanorod tip. The lattice fringes of $\left\{\begin{array}{lll}0 & 0 & 2\end{array}\right\}$ basal planes of the nanorod exhibit a cup-like shape, and the nanorod consists of such stacking cones. Nanosized voids exist in the centre of the $\mathrm{BN}$ nanorods as a structural feature [11-13]. Positive curvatures are evident in the vicinities of the cone apexes and non-hexagonal member rings must exist
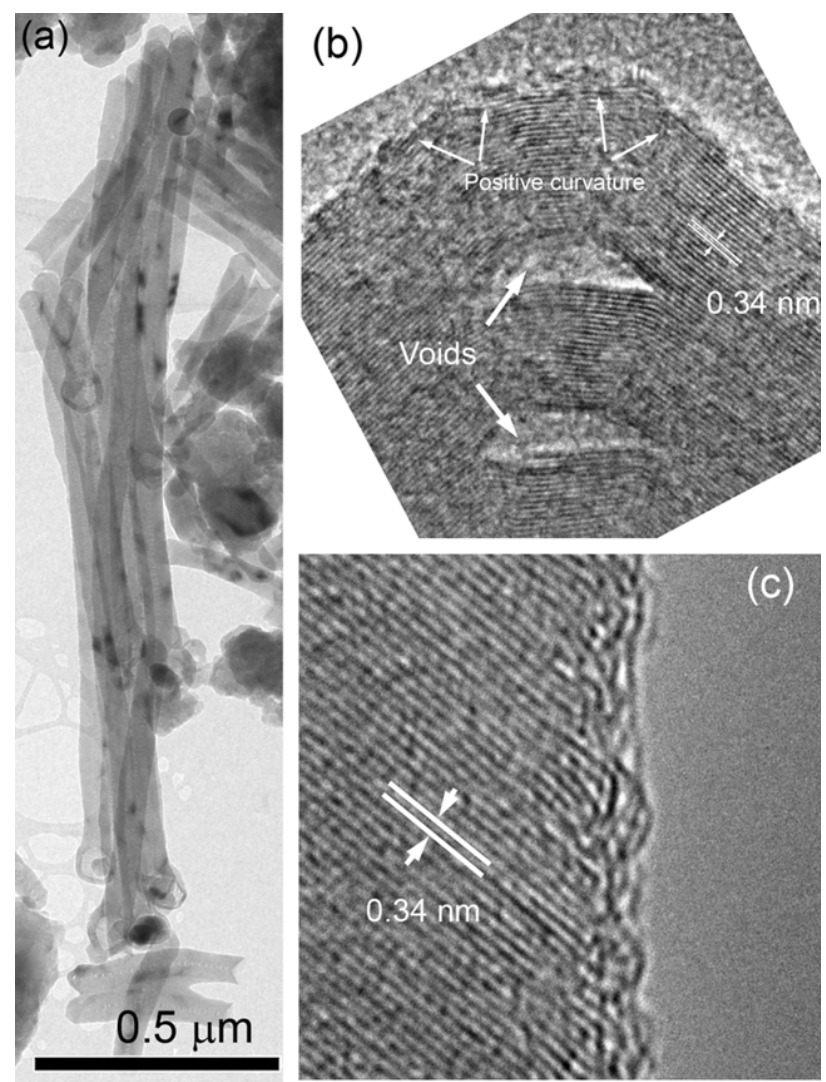

Figure 2. (a) Bright-field TEM image reveals the typical morphology of the nanorods: conical tips and attached catalyst particles. (b) A lattice image of a nanorod tip displays nanosized voids at the centre of the nanorods and a stacking cone structure is evident. (c) A lattice image of the side morphology of a nanorod shows the cones interweave with each other on the nanorod side surface.

in the hexagonal network of the basal planes so as to generate the curvatures and thus form the cones [14]. Adjacent cones interweave with one another on the side surfaces of the nanorod (figure 2(c)), which indicates a significant portion of the bonds near the side surfaces may be $\mathrm{sp}^{3}$ hybridized [15].

$\mathrm{FE}$ characteristics of the $\mathrm{BN}$ nanorods were measured by applying a voltage $V$ increasing from $\sim 1$ to $\sim 3 \mathrm{kV}$ with a step of $60 \mathrm{~V}$. The sample-anode distance $d$ was fixed at $100 \mu \mathrm{m}$. The emission current $I$ is shown in figure $3(a)$ as a function of the applied electric field $E$ in both logarithmic and linear scales referring to the left and the right vertical axes, respectively. The turn-on field $E_{\text {to }}$, which is defined arbitrarily as the macroscopic field $\left(E_{\mathrm{mac}}=V / d\right)$ for which a $10 \mu \mathrm{A} \mathrm{cm}^{-2}$ emission current density is measured [16], is estimated to be $\sim 28 \mathrm{~V} \mathrm{\mu m}^{-1}$ (the area of the substrate is $\sim 1 \mathrm{~cm}^{2}$ ) (It is worth mentioning that different definitions of $E_{\mathrm{to}}$ are found in the literatures; for example, the $E_{\mathrm{to}}$ of the

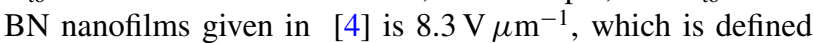
however as the field at the emission current of $0.1 \mathrm{nA}$. Such

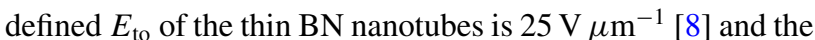
present sample has a value of $<10 \mathrm{~V} \mu \mathrm{m}^{-1}$ if this definition is adopted.) It is about $5-10$ times higher than those of the carbon nanotubes [16]. The large $E_{\text {to }}$ of the present sample might be due to the morphology effect. It is well known that the local field $E_{\text {loc }}$ at the emitting surface can be enhanced as 

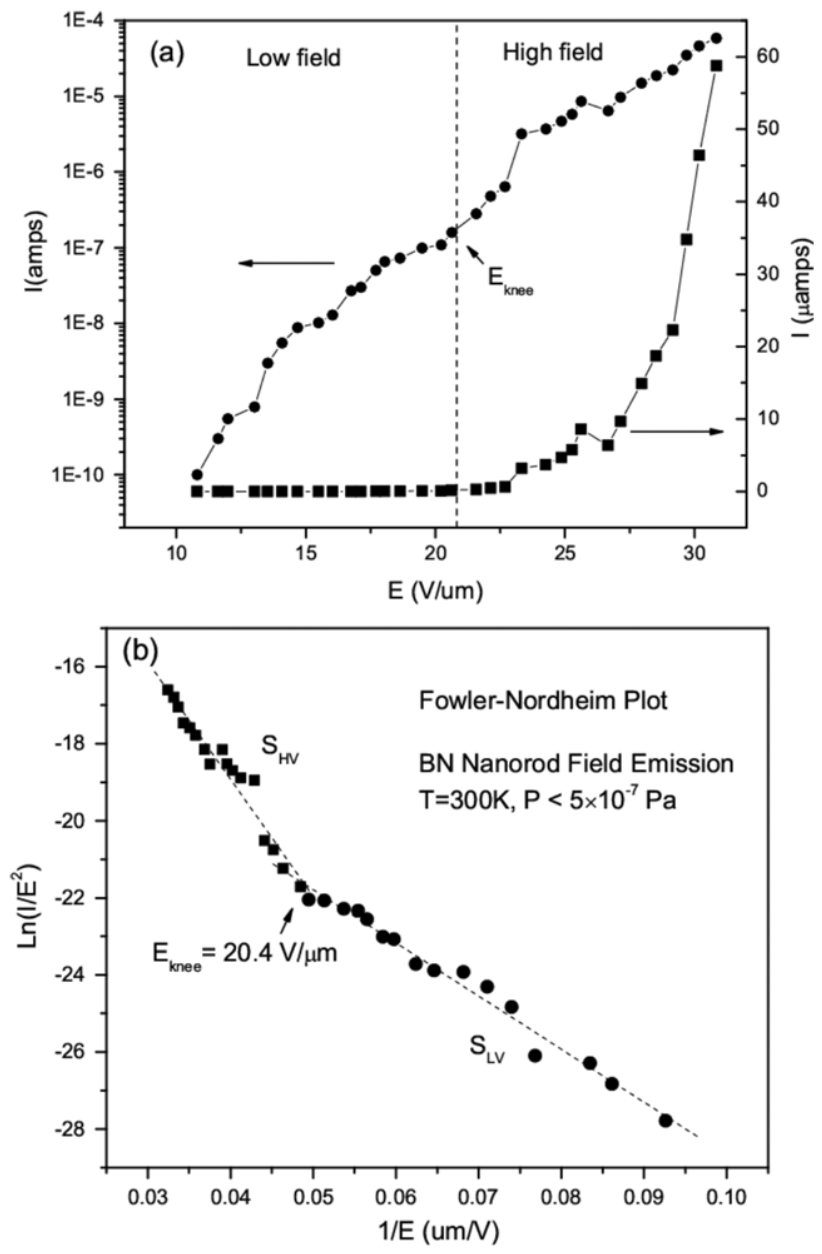

Figure 3. (a) Emission current $(I)$ as a function of the applied electric field $(E)$ both in logarithmic and linear scales. $(b)$ The FN plot of the FE characteristics shows two distinct slopes corresponding to low and high voltages.

the size of the emitter is reduced [19]. For a given applied voltage $V$, a field conversion factor $\beta$ can be introduced as $E_{\mathrm{loc}}=\beta \cdot V=\beta d \cdot E_{\mathrm{mac}}$ to illustrate the enhancement. To explicitly show the size of the emitter, some authors relate $E_{\text {loc }}$ to $V$ as $E_{\text {loc }}=V /\left(\alpha^{\prime} \cdot r\right)=\left(d / \alpha^{\prime}\right)\left(E_{\text {mac }} / r\right)$, where $r$ is the radius of the emitter tip and $\alpha^{\prime}$ is the enhancement factor depending on $d$ and $r$. For an ideal isolated spherical emitter where $d \gg r$, the local field is reciprocally proportional to the emitter size [19]. In general, the smaller the emitter tip, the lower the applied voltage and the lower the macroscopic field required to reach a certain local field that modifies the surface barrier for measurable electron penetration. However, since the turn-on field is defined as a macroscopic field relating to the emission current density, the $E_{\text {to }}$ of the nanorods also depends on their coverage on the substrate. A fraction of the silicon surface has fewer nanorods which can also increase the $E_{\text {to }}$ as the emission area is overestimated and the current density is underestimated.

Fowler-Nordheim (FN) theory has been commonly practiced to explain the FE characteristics of different emitters, while the theory is derived from and strictly applicable to metal surfaces at high fields and low temperatures [20]. FE of these FN emitters is related to electron tunnelling through an

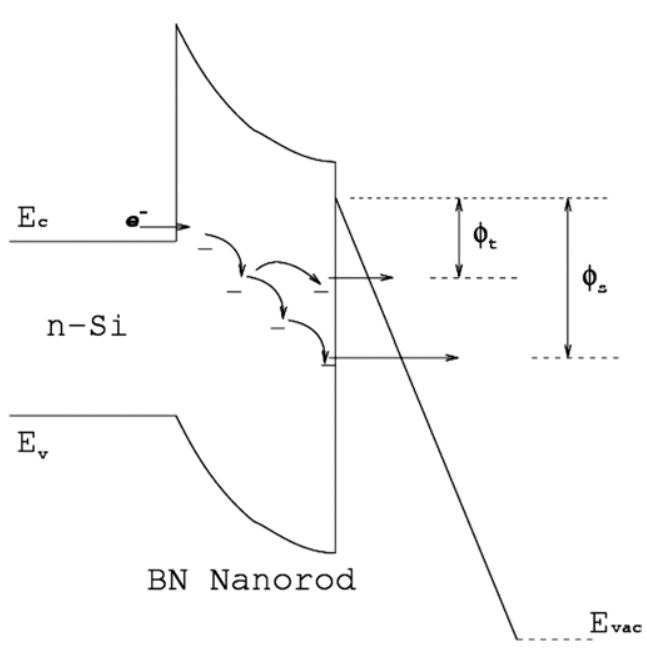

Figure 4. An illustrative sketch of the energy-band diagram of a BN nanorod showing the shallow and deep levels.

interface barrier. According to FN theory, the FE current $i$ from an emitter of tip radius $r$ is related to the applied electric field $E$ by the FN equation

$$
i(r)=a(r) \cdot K_{1} \frac{\beta^{2}(r) \cdot E^{2}}{\phi} \exp \left[-\frac{K_{2} \cdot \phi^{3 / 2}}{\beta(r) \cdot E}\right]
$$

where $a(r)$ is the emitting area, $\phi$ is the work function, $\beta(r)$ is the field conversion factor and $K_{1}$ and $K_{2}$ are constants. The linearity of the FN plot (i.e. $\ln \left(I / E^{2}\right)$ versus $1 / E$ ) is an indication of the FN emission, and equation (1) shows that the slope of a FN plot, $S_{\mathrm{FN}}=-K_{2} \cdot \phi^{3 / 2} / \beta(r)$, is unique for an emitter of specific geometry and work function when the measurement is conducted with a constant sample-anode distance. Figure $3(b)$ is the FN plot of the BN nanorods. However, two distinct FN slopes, i.e. $S_{\mathrm{LV}}$ and $S_{\mathrm{HV}}$, are observed for low and high electric fields, and the electron emission of the nanorods cannot be explained by FN theory.

The energy-band diagram of the silicon substrate and the $\mathrm{BN}$ nanorod is used to consider the electron emission mechanism. The illustrative band diagram is shown in figure 4, which is similar to that of $\mathrm{BN}$ nanofilms [4]. Cathodoluminescence (CL) studies [12] show that shallow donors-deep acceptors and deep-level features exist in the nanorod band gap arising from the non-hexagonal member rings near the centres of the nanorods [9] and the $\mathrm{sp}^{3}$ features on their side surfaces, respectively. It indicates that the shallow and deep levels exhibit site-related features, i.e. they populate at the tip-vacuum and side-vacuum interfaces, respectively. When a bias was applied, electrons first tunnel through the interface barrier between the silicon substrate and the $\mathrm{BN}$ nanorod to these defect levels, electron transport in the BN nanorod is through hopping conduction [18], and the electrons then emit to the vacuum from both types of defect levels. The height of the effective barrier of the shallow-level related emission at tip-vacuum interfaces $\phi_{t}$ is smaller than that of deep-level related emission at side-vacuum interfaces $\phi_{\mathrm{s}}$ (figure 4). Moreover, the field conversion factor for tips $\beta_{\mathrm{t}}(r)$ is larger than that for side surfaces $\beta_{\mathrm{s}}(r)$ for a given radius $r$ 
[19]. The analysis suggests that the FE of the present sample is from an ensemble consisting of the two types of emitters (i.e. the tip and the side surfaces) of varying sizes (figure $1(b)$ ), and the total emission current of the sample $I_{t}$ can be approximately written as [21]

$$
I_{\mathrm{t}}=\sum_{r}\left[G_{\mathrm{t}}(r) \cdot \mathrm{i}\left(r, \phi_{\mathrm{t}}\right)+G_{\mathrm{s}}(r) \cdot \mathrm{i}\left(r, \phi_{s}\right)\right],
$$

where $G_{\mathrm{t}, \mathrm{s}}(r)$ is the number of tips (side surfaces) having radii between $r-\Delta r$ and $r+\Delta r$, which can be extracted from the histogram (figure $1(b)$ ). However, the emitting area of the nanorod side surface $a_{\mathrm{s}}$ overwhelms that of the nanorod tip $a_{\mathrm{t}}$, i.e. $a_{\mathrm{s}} \gg a_{\mathrm{t}}$ for a given $r\left(a_{\mathrm{s}} / a_{\mathrm{t}} \sim\right.$ the aspect ratio of $\left.10^{2}-10^{3}\right)$. The random orientation of the nanorods results in $G_{\mathrm{s}}(r)>G_{\mathrm{t}}(r)$. By defining a total emission area for each type of emitters $A_{\mathrm{t}, \mathrm{s}}=\sum_{r} G_{\mathrm{t}, \mathrm{s}}(r) a_{\mathrm{t}, \mathrm{s}}(r)$, we have $A_{\mathrm{s}} \gg A_{\mathrm{t}}$ (s and $\mathrm{t}$ stands for side surface and tip, respectively). It has been found that a Gaussian distribution of tip sizes can modify the FN slopes because the value of tip radius $r$ making the peak contribution to the total emission current varies with the applied voltage $V$ [21]. Similarly, the $S_{\mathrm{LV}}$ shown in figure $3(b)$ corresponds to the tip emission, which has a large field conversion factor and a low barrier height but a small total emission area. As the applied voltage increased, the side-surface emission contributes to the total emission current dominantly since they are dominant in the emission area, and the FN plot changes slope to $S_{\mathrm{HV}}$ accordingly. Furthermore, as the emission from the side surfaces started, the electrons hopping to the tips, i.e. to the shallow levels, are reduced, so the electron emission from the tips might be saturated or even annihilated and a clear switchover electric field $E_{\text {knee }}$ therefore appears (figure $3(b)$ ).

The deviation of the FN plot from linearity has been observed in many nanoemitters [17,23]. Several mechanisms have been proposed for the deviation; for example, vacuum space charge at high-current density can reduce the local electric field surrounding the emitter and thus results in the saturation of the emission current at high field [22]. The space-charge related saturation builds up gradually at a certain field and the emission current no longer increases. It is thus not applicable to the $\mathrm{BN}$ nanorods since both phenomena have not been spotted from the emission of the BN nanorods. The adsorption effect might also be responsible for the deviation and a gradual adsorption or desorption of the adsorbates on the emitter surface causes a smooth change in the FN slope [23]. Chen et al [24] found that the bending down of the surface band can be enhanced as the emitter temperature decreases, resulting in the reduction of the emission current, while no significant variation in the temperature of the $\mathrm{BN}$ nanorods was observed during the emission measurement. In addition, the anomalous rise in the emission current from carbon nanotubes at low applied fields can be attributed to interactions between neighouring nanotube tips [17] where the FN slope at a high field was smaller than that at a low field. Nevertheless, there might be some other unidentified physical processes accounting for the observed FE characteristics of the BN nanorods, and further experimental and theoretical work should be conducted to verify the proposed mechanism.

\section{Conclusions}

In conclusion, we measured the $\mathrm{FE}$ characteristics of $\mathrm{BN}$ nanorods grown on an $n$-Si substrate. Electrons were emitted from two types of defect levels, shallow donor and deep levels, in the band gap of the nanorods, which populated near the tips and the side surfaces of the nanorods, respectively. The switchover from the tip emission to side emission results in two distinct FN slopes. It is suggested that well-aligned conical nanorods might improve the FE characteristics of the BN nanorods.

\section{Acknowledgments}

The authors thank Dr Sally Stowe, Dr Cheng Huang and Ms Lily Shen for assistance with the electron microscopes. They are grateful to Dr John Fitz Gerald for assistance with the electron microscope and his valuable discussions. This work is financially supported, in part, by the Australian Research Council under the Nanotube Programme of the Centre of Functional NanoMaterial.

\section{References}

[1] Powers M J, Benjamin M C, Portor L M, Nemanich R J, Davis R F, Cuomo J J, Doll G L and Harris S J 1995 Appl. Phys. Lett. 67, 3912

[2] Pryor R W 1996 Appl. Phys. Lett. 681802

[3] Sugino T, Kawasaki S, Tanioka K and Shirafuji J 1997 Appl. Phys. Lett. 712704

[4] Sugino T, Kimura C and Yamamoto T 2002 Appl. Phys. Lett. 803602

[5] Jayatissa A H, Sato F, Saito N, Sawada K, Masuda T and Nakanishi Y 1999 J. Vac. Sci. Technol. B 17237

[6] Kimura C, Yamamoto T, Hori T and Sugino T 2001 Appl. Phys. Lett. $\mathbf{7 9} 4533$

[7] DeHeer W A, Chatelain A and Ugarte D 1995 Science 2701179

[8] Cumings J and Zettl A 2004 Solid State Commun. 129661

[9] Azevedo S, Mazzoni M S C, Chacham H and Nunes R W 2003 Appl. Phys. Lett. 822323

[10] Chen Y, Zou J, Campbell S J and Le Caer G 2004 Appl. Phys. lett. 842430

[11] Zhang H Z, FizGerald J D, Yu J and Chen Y 2006 J. Am. Ceram. Soc. 89675

[12] Zhang H Z, Phillips M, FizGerald J D, Yu J and Chen Y 2006 Appl. Phys. Lett. 88093117

[13] Zhang H Z, FizGerald J D, Chadderton L T, Yu J and Chen Y 2006 Phys. Rev. B $\mathbf{7 4} 045407$

[14] Ebbesen T W 1998 Acc. Chem. Res. 31558

[15] Collazo-Davila C, Bengu E, Marks L D and Kirk M 1999 Diamond Relat. Mater. 81091

[16] Wang Z L and Hui C 2003 Electron Microscopy of Nanotubes (Norwell: Kluwer) p 191

[17] Collins P G and Zettl A 1997 Phys. Rev. B 559391

[18] May P W, Höhn S, Wang W N and Fox N A 1998 Appl. Phys. Lett. 722182

[19] Marcus R B, Chin K K, Yuan Y, Wang H and Carr W N 1990 IEEE Trans. Electron Devices 371545

[20] Gomer R 1961 Field Emission and Field Ionization (Cambridge: Harvard University Press)

[21] Harvey R J, Lee R A, Miller A J and Wigmore J K 1991 IEEE Trans. Electron Devices $\mathbf{3 8} 2323$

[22] Dyke W P and Dolan W W 1956 Adv. Elect. Electron Phys. 8 89

[23] Zhang H Z, Wang R M and Zhu Y W 2004 J. Appl. Phys. 96624

[24] Chen S Y and Lue J T 2002 New J. Phys. 479.1 